\title{
Revisiting the peculiar regional distribution of muscle fiber types in rat Sternomastoid Muscle
}

\author{
Barbara Ravara (1,2,3), Valerio Gobbo (4), Damiana Incendi (5), Andrea Porzionato \\ (2,5), Veronica Macchi (5), Raffaele De Caro (5), Dario Coletti (6,7,8), Tiziana \\ Martinello (2,9), Marco Patruno (2,9)
}

(1) Laboratory of Translational Myology, Department of Biomedical Sciences, University of Padova, Italy; (2) Interdepartmental Research Center of Myology (CIR-Myo), University of Padova, Italy; (3) A\&C M-C Foundation for Translational Myology, Padova, Italy; (4) CNR Institute of Neuroscience, Padova, Italt; (5) Human Anatomy Section of the Department of Neurosciences, University of Padova, Italy ; (6) Sorbonne Universités, UPMC Univ Paris 06 (CNRS, UMR 8256, INSERM ERL U1164), Institut Biologie Paris-Seine, Paris, France; (7) Departmrent of Anatomy, Histology, Forensic Medicine \& Orthopedics, School of Medicine Sapienza University of Rome, Italy; (8) Interuniversity Institute of Myology, Italy; (9) Department of Comparative Biomedicine and Food Science, University of Padova, Italy.

This article is distributed under the terms of the Creative Commons Attribution Noncommercial License (CC BY-NC 4.0) which permits any noncommercial use, distribution, and reproduction in any medium, provided the original author(s) and source are credited.

\begin{abstract}
The sternomastoid (SM) muscle in rodents is known to have a peculiar distribution of fiber types with a steep gradient from surface to deep region. We here further characterize this peculiar regional distribution by quantitative histochemical morphometrys. In Hematoxylin-Eosin (H-E) stained transverse cryosections harvested in the medial portion of the muscle we counted around 10.000 myofibers with a mean diameter of $51.3 \pm 12.6(\mu \mathrm{m})$. Cryisections of the SM stained by SDH reaction clearly show two distinct regions, toward the deep surface of the muscle a $40 \%$ area that contains packed SDH-positive myofibers, while the remaining area of the SM toward the external surface presents a more checker-board appearance. On the other hand, in the deep region of SM type 1 (slow contracting) muscle fibers, caracterized by positive acidic ATPase $\mathrm{pH}$ 4.35 reaction, are only the $24.5 \%$ of the fibers in the deep area of SM muscles, being restricted to the deepest region. The $75.5 \%$ of the myofibers in the deep region are of the fast contracting types (either $48.4 \%$ 2A, SDH -positive fibers or $27.1 \%$ 2B, SDH-negative fibers, respectively). As expected the 2B muscle fibers, acidic ATPase $\mathrm{pH}$ 4.3-negative and SDH-negative, present the largest size, while Type 1 fibers, acidic ATPase $\mathrm{pH}$ 4.3-positive and SDH-positive, present the smallest size in rat SM muscle. Based on present and previous observations, comparison of change in absolute number and/or percentage of the fiber types in any experimental model of muscle atrophy/hypertrophy/plasticity/pathology/recovery in the rat SM, and possibly of all mammals, will ask for morphometry of the whole muscle cross-sections, muscle sampling by bioptic approches will provide only comparable data on the size of the different types of muscle fibers.
\end{abstract}

Key Words: rat, Sternomastoid muscle, muscle fiber types, SDH, myofibrillar ATPases, regional distribution

Eur J Transl Myol 28 (1): xxx-xxx, 2018

The sternomastoid muscle (SM) in rodents is known to have a peculiar distribution of fiber types with a steep gradient from surface to deep region. ${ }^{1}$ This behavior is also present in leg muscles (e.g., tibialis anterior muscle), but not at extent of the SM muscle. ${ }^{2-4} \mathrm{We}$ are characterizing in normal animals this peculiar regional distribution by quantitative histochemical analyses of transverse sections in the third medial portion of the muscle. We will present results suggesting that comparison of change in absolute number and/or percentage of the fiber types in the rat SM will ask for morphometry of the whole muscle cross-section, while muscle bioptic samplings will provide only consistent results on size of muscle fibers. 


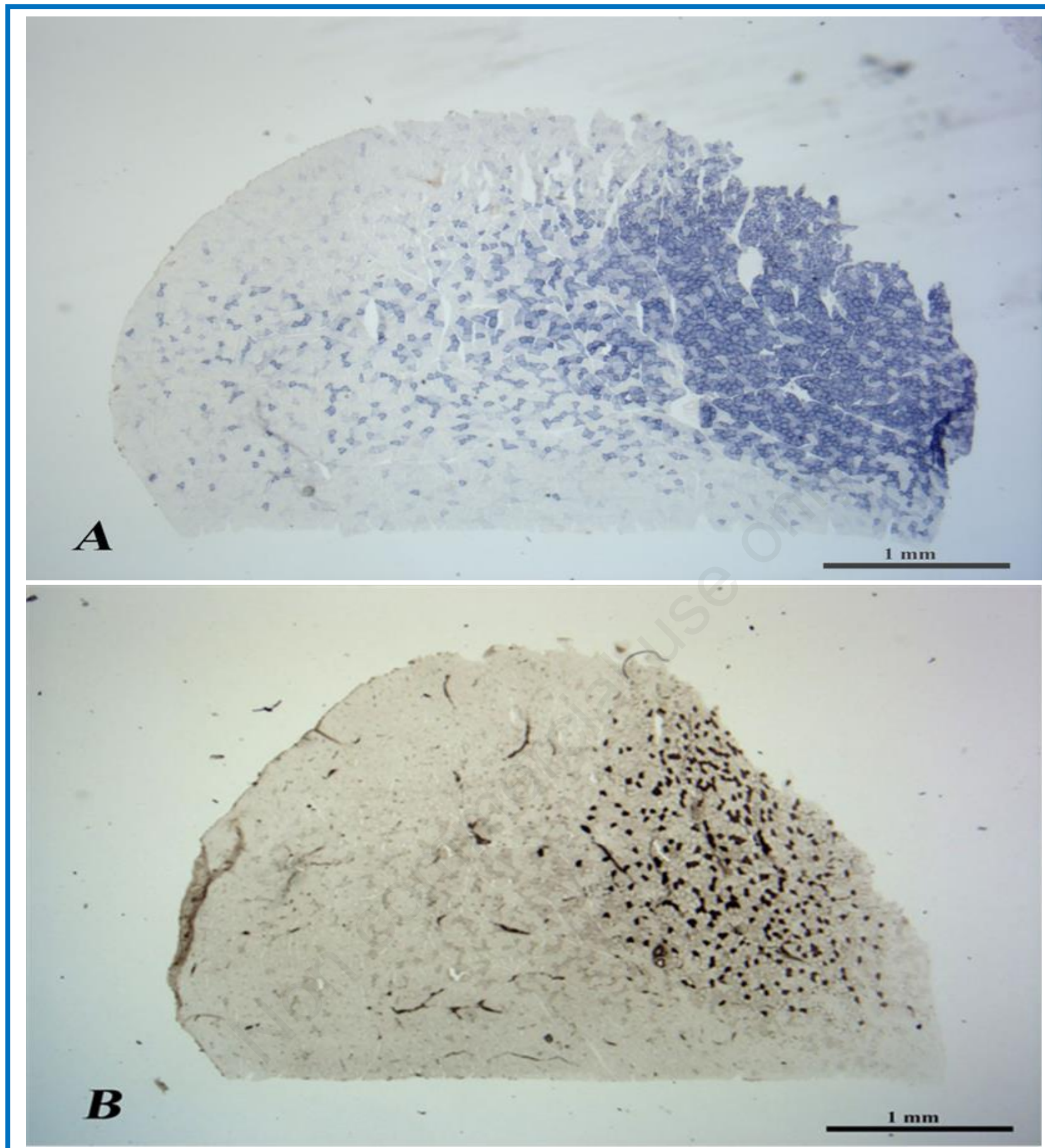

Fig 1. Transverse cross-section of normal rat SM muscle. Panel A, SDH reaction. In the deep region are present small diameter, intense blue fibers, while the superficial region presents higher diameter unstained fibers (type 2B) and some ligth to midle blue fibers (Type 2A). Panel B, ATPase pH 4.35 reaction. The black fibers (type1) are restricted to deepest region of the SM muscle. Scale Bar $1 \mathrm{~mm}$.

\section{Materials and Methods}

\section{Animals and harvesting of muscles}

Adult females Wistar rats, $300 \mathrm{~g}$ of weight were used. These animals come from the vivarium of the Department of Neurosciences - University of Padova.
The animals were kept in cages with light/dark cycle of $12 \mathrm{~h}$, room temperature $(24 \pm 2)$, food and water $a d$ libitum. All procedures in this study were according to the international ethical principles of animal experimentation and approved by the Ethics on Animal Experimentation Committee (EAEC) of University of 
Table 1. Mean myofiber diameter $(\mu m)$ in the SM rat muscle stained by $H$-E. Values are mean $\pm S D$
All muscle fibers
SM dx
SM sx

$51.6 \pm 26.7$

$50.9 \pm 12.1$

Mean of two SM

$51.3 \pm 12.7$

Total fibers in Deep and Superficial region 9981

Padova. Animals were killed by deep anaesthesia and muscles were excised, weighted, frozen in liquid nitrogen $\left(-196^{\circ} \mathrm{C}\right)$ and kept at $-80^{\circ} \mathrm{C}$ until use.

Histology and histochemistry of the sternomastoid muscle

Size, and absolute or percent contents of different muscle fiber types were determined in transverse cryosections of the SM muscle harvested in the medial third of the muscle. Serial $10 \mu \mathrm{m}$ cross-sections were cut on a cryostat microtome at $-25^{\circ} \mathrm{C}$, mounted on slides and stained in small glass jars. From stained sections digital images were collected at magnification of x 2.5 and x 10 using a transmission light microscope mounting a HDMI camera. Quantitative evaluations were performed on the collected pictures using Scion Image for Windows version Beta 4.0.2 (2000 Scion Corporation). Muscle fiber sizes and regional distribution of the muscle fiber type were determined using the Adobe Photoshop software (Adobe Systems Incorporated, San Jose, CA).

\section{Absolute number of muscle fibers in rat SM muscle}

To determine the absolute number of muscle fibers in the rat SM muscle, cryosections were stained with standard Hematoxylin-Eosin (H-E) procedure. ${ }^{5}$

Fiber typing of the muscle fibers was performed by the following histochemical methods

\section{Succinate Dehydrogenase (SDH) reaction}

SDH reaction was performed to distinguish between oxidative, "less" oxidative and non-oxidative muscle fibers. Muscle sections were incubated in SDH incubation solution for $60 \mathrm{~min}$ a $37^{\circ} \mathrm{C}$. Sections were rinsed in distilled $\mathrm{H} 2 \mathrm{O}, 3 \times 1 \mathrm{~min}$ and removed unbound NBT from sections with $3 \times 1$ min exchanges of acetone solutions $30,60,90 \%$ in distilled $\mathrm{H} 2 \mathrm{O}$, in increasing then decreasing concentrations. Sections were rinsed in distilled $\mathrm{H} 2 \mathrm{O}, 3 \times 1 \mathrm{~min}$ and dehydrated in graded alcohol solutions (ethanol 70, 90, 100\%) cleared in xylene and mounted in permanent medium: Canada balsam. SDH incubating solution: NBT (nitro blue tetrazolium): $10 \mathrm{mg}$; SDH stock solution: $10 \mathrm{ml}$. Adjust to $\mathrm{pH} 7.2$ to 7.6 with $0.1 \mathrm{~N} \mathrm{HCl}$ or $\mathrm{NaOH}$. SDH stock solution: $0.2 \mathrm{M}$ sodium succinate solution 100ml, 0.2M Phosphate buffer, $\mathrm{pH} 7.4$ $100 \mathrm{ml}$, make freshly at least every two weeks.

Histochemistry by Myofibrillar actomyosin ATPase

Two different procedures were used for staining myofibrillar actomyosin ATPase using essentially the same methods described by Brooke and Kaiser, ${ }^{6,7}$ and by Guth and Samaha. ${ }^{8-10}$ For determination of the alkali preincubated myofibrillar ATPase activity the following procedures were employed. cryosections were air dried for $10 \mathrm{~min}$ a room temerature and fixed for $3 \mathrm{~min}$ in $5 \%$ $(\mathrm{w} / \mathrm{v})$ formaldehyde in $200 \mathrm{mM}$ sodium cacodylate, $68 \mathrm{mM} \mathrm{CaCl} 22 \mathrm{H} 2 \mathrm{O}$ and $340 \mathrm{mM}$ sucrose (adjusted with $\mathrm{HCl}$ to $\mathrm{pH} 7.6$ before adding the formaldehyde).After washing in wash solution $100 \mathrm{mM}$ Tris, $18 \mathrm{mM} \mathrm{CaCl} 2$ (pH 7.8), an alkaline pre-incubation was performed for $15 \mathrm{~min}$ a room temperature in a solution containing 200mM 2-amino-3-methyl-1 propanol (adjusted with $\mathrm{HCl}$ to $\mathrm{pH}$ 10.4). After two successive 1 min incubation in the wash solution, sections were incubated for $45 \mathrm{~min}$ a $37^{\circ} \mathrm{C}$ in ATP solution $(2,7 \mathrm{mM} \mathrm{ATP,} 90 \mathrm{mM} \mathrm{CaCl} 2$, $100 \mathrm{mM}$ sodium barbital adjusted with $\mathrm{NaOH}$ to $\mathrm{pH} 9.4$ ). For the acid preincubation myofibrillar ATPase activity $(10,11)$, the following procedures were utilized. Sections were air dried for $10 \mathrm{~min}$ at room temperature and incubated in a solution consisting of $100 \mathrm{mM}$ sodium acetate (adjusted with $\mathrm{HCl}$ to $\mathrm{pH} 4.35$ ) for $10 \mathrm{~min}$ a room temperature. After washing $2 \times 1 \mathrm{~min}$ in $18 \mathrm{mM} \mathrm{CaCl} 2$ and $100 \mathrm{mM}$ Tris $\mathrm{HCl} \mathrm{pH} \mathrm{7.8,} \mathrm{the} \mathrm{sections} \mathrm{were} \mathrm{incubated} \mathrm{a}$ $37^{\circ} \mathrm{C}$ for $45 \mathrm{~min}$ in ATP solution together to serial sections with alkali preincubation. After washing $2 \times 30$ sec in distilled $\mathrm{H} 2 \mathrm{O}$, sections were incubated for $5 \mathrm{~min}$ in $2 \%(\mathrm{w} / \mathrm{v}) \mathrm{CoCl} 2$, washed $2 \times 30 \mathrm{sec}$ in distilled $\mathrm{H} 2 \mathrm{O}$, sections were incubated $5 \mathrm{~min}$ in $2 \%(\mathrm{v} / \mathrm{v})(\mathrm{NH} 4) 2 \mathrm{~S}$, washed in distilled $\mathrm{H} 2 \mathrm{O} 2 \times 30 \mathrm{sec}$, and dehydrated in graded alcohol solution (ethanol 70-90-100\%) cleared in xilene, and mounted with Canada balsam medium.

\section{Results}

Morphometry: number and size of rat SM muscle fibers in H-E stained cryosections.

In the rat SM muscle cryosections harvested in the medial portion of the muscle we counted around 10.000 
myofibers. The mean diameter in these muscle sections stained by $\mathrm{H}-\mathrm{E}$ was $51.3 \pm 12.7 \mu \mathrm{m}$ (Table 1 ).

Histochemistry of sternomastoid rat muscle

Fiber typing by SDH reaction

In Fig.1A is presented a transverse section from the medial region of a rat $\mathrm{SM}$ muscle stained by $\mathrm{SDH}$ reaction. The cryosection clearly shows two distinct regions, a right part of the section that cover around $40 \%$ area that contains SDH positive myofibers (right part of Fig.1A) and a the left part of Fig.1A; the latter encopasses the other $60 \%$ area of the SM with a more checker-board appearance. The rigth part of the cryosection, peculiarly rich in SDH positive muscle fibers, corresponds to the deep part of the SM, while the left part of the figure correspond to the superficial region of the SM. Table 2 shows that SDH-positive myofibers are the $72.9 \%$ of the total myofibers of SM deep region. In the superficial region (left part of the cryosection) based on $\mathrm{SDH}$ staining (Fig.1A), the mitochondrial rich, 2A muscle fibers are $31.1 \%$. Since very few or none of type 1 muscle fibers are present there (see ATPase histochemistry reaction). The $68.9 \% 2 \mathrm{~B}$ negative fibers are that of the myofibers present in this region of the SM muscle. Figure 2 shows six rappresentative fields at highter magnification taken from periferal, intermediate, and deep portions of cryosections stained either by SDH (A, B, C) or acidic ATPase (D, E, F).

Histochemistry by Myofibrillar actomyosin ATPase In the deep region (right part of Fig.1B), the type 1 (slow contracting) muscle fibers, identified by positive acidic ATPase $\mathrm{pH} 4.35$ (Fig.1B), are only the $24.5 \%$ of the fiber of this part of SM muscle (Table2). On the other hand, the slow type muscle fibers, i.e., those positive at the acidic ATPase $\mathrm{pH} 4.35$ are restricted in the deepest region of SM muscle. Thus, $75.5 \%$ of the myofibers in the deep region are of the fast contracting types (either $48.4 \% 2 \mathrm{~A}$ and $27.1 \%$ are $2 \mathrm{~B}$, respectively).

In the superficial region, left part of the cryosection, based on acidic ATPase $\mathrm{pH} 4.35$, the type 1 positive fibers are absent, the type $2 \mathrm{~A}$ and muscle fibers characterized by an intermediate intensity decrease to $20.3 \%$. Therefore the vast majority of the muscle fibers present in the superficial region of the SM muscle are of the $2 \mathrm{~B}$ type $(68.9 \%$ as SDH- negative fibers or $79.7 \%$ when counted in the acidic ATPase $\mathrm{pH} 4.35$ ).

Fiber size of the three different fiber types of the SM muscle

As expected, Table 1, shows that the acidic ATPase $\mathrm{pH}$ 4.35 negative and SDH-negative $2 \mathrm{~B}$ type myofibers, present the largest size among the three types of muscle fiber of rat SM muscle. In the superficial region based on acid ATPase $\mathrm{pH} 4.35$, the $2 \mathrm{~B}$ fiber size result $47.6 \mu \mathrm{m}$ and the $2 \mathrm{~A}$ fiber $35.3 \mu \mathrm{m}$. In the deep region the fiber size of 1 fibers (slow contracting) result to be the smallest: $29.2 \mu \mathrm{m}$; while the $2 \mathrm{~A}$ and $2 \mathrm{~B}$ fiber size are $36.8 \mu \mathrm{m}$ on average in size. Based on mitochondrial enzyme activities, SDH reaction, in the superficial region the size of $2 \mathrm{~B}$ fiber recognizable as the larger ones are $53.3 \mu \mathrm{m}$ and in the deep region $41.1 \mu \mathrm{m}$, while the 1 and $2 \mathrm{~A}$ fiber size in the superficial region and in the deep region is respectively $35.8 \mu \mathrm{m}$ and $33.8 \mu \mathrm{m}$.

\section{Discussion}

The peculiarity of the SM rat muscle is the presence of two distinct regions, where the fiber type composition is characterized in both zone by $2 \mathrm{~B}$ fibers that are easily

Table 2. Mean myofiber diameter $(\mu \mathrm{m})$ in deep and superficial region of rat SM stained by SDH or ATPase, pH 4.35 reactions. Values are mean $\pm S D$.

\section{SDH reaction}

\begin{tabular}{lcc}
\hline & Deep region & Superficial region \\
\hline & & \\
Type fibers 1 + 2A & $33.8 \pm 7.5$ & $35.8 \pm 9.2$ \\
Type fibers 2B & $41.1 \pm 9.2$ & $53.3 \pm 10.8$ \\
Total number fibers & 5211 & 3975 \\
\hline
\end{tabular}

ATPase pH 4.35

\begin{tabular}{lccc}
\hline & Deep region & & Superficial region \\
\hline Type fibers 1 & $29.2 \pm 6.5$ & Type fibers 2A & $35.3 \pm 8.5$ \\
Type fibers 2A $+2 B$ & $36.8 \pm 9.2$ & Type fibers 2B & $47.6 \pm 11.9$ \\
Total number fibers & 5802 & & 4179
\end{tabular}


Table 3. Number and percentage of SM fiber types in deep or superficial regions by SDH or ATPase pH 4.3 SDH reaction

Deep region Superficial region

Type fibers $1+2 \mathrm{~A}$

Number

Percentage

3800

$72.9 \%$

1411

Number

Percentage

Total number fibers

Total fibers Deep plus Superficial region
1235

$31.1 \%$

2740

$68.9 \%$

3975

9186

ATPase pH 4.35

\begin{tabular}{lccc}
\hline & Deep region & Superficial region \\
\hline Type fibers 1 & 1422 & Type fibers 2A & \\
Number & $24.5 \%$ & & 849 \\
Percentage & & Type fibers 2B & $20.3 \%$ \\
Type fibers 2A+2B & 4380 & & 3330 \\
Number & $75.5 \%$ & & $79.7 \%$ \\
Percentage & 5802 & & 4179 \\
Total number fibers & 9981 & & \\
Total fibers Deep and Superficial region & & \\
\end{tabular}

recognizable from their major size, while the 1 and $2 \mathrm{~A}$ are the smallest. The SM muscle presents a very heterogeneous distribution of muscle fiber types with a large predominance of the type $2 \mathrm{~B}$ in the superficial region while moving to deep regions there is a progressive enrichment of 2A fibers. Only in the deepest region of the SM muscle there are type 1 muscle fibers (Fig 1B and Table 3).

It remains to be determined if the content of fiber types varies along the muscle length and if applying new imaging processing as the Machine-learning algorithms (Deep Neural Networks) that have been proved to be very powerful methods for automatic image segmentation, especially in the field of histological analyses, ${ }^{11}$ some of the probleams we are facing with non homogeneous muscles would be overcommed. Anyhow, as demonstrated here and by previously, ${ }^{1}$ in the rat SM muscle comparative analyses for experimental models of muscle plasticity, in normal and diseased muscles, ${ }^{12-26}$ will require morphometry of the whole cross-sections. In conclusion, in case of bioptic fragments of SM muscle, in small, but conceivably also large, mammals, only the size of the different fiber types could be evaluated to avoid systematic errors related to diferent regional sampling.

\section{List of acronyms}

ATPase - myofibrillar Actomyosin ATPase histochemistry

EAEC - Ethics Committee on Animal Experimentation

H-E - Hematoxylin-Eosin reaction

SDH - succinate dehydrogenase reaction

SM - sternomastoid muscle

\section{Author's contributions}

Authors equally contributed to the manuscript.

\section{Acknowledgments and Funding}

BR thanks for support A\&C M-C Foundation for Translational Myology, Padova, Italy.

\section{Conflict of Interest}

The authors declare no conflicts of interests.

\section{Ethical Publication Statement}

We confirm that we have read the Journal's position on issues involved in ethical publication and affirm that this 


\section{Regional distribution of muscle fiber types in rat sternomastoid muscle}

Eur J Transl Myol 28 (1): 117-123, 2018

typescript is consistent with those guidelines.

\section{Corresponding Author}

Barbara Ravara, Laboratory of Translational Myology, of the Iterdepartmenta Research Center for Myology (CIRMyo), c/o Department of Biomedical Sciences, University of Padova and A\&C M-C Foundation for Translational Myology, Padova, Italy.

E-mail: barbara.ravara@unipd.it

\section{E-mails of co-authors}

Damiana Incendi: damiana.incendi@unipd.it Andrea Porzionato: andrea.porzionato@unipd.it Valerio Gobbo: gobbov@bio.unipd.it Veronica Macchi: veronica.macchi@unipd.it Raffaele De Caro: Raffaele.decar@unipd.it Dario Coletti: dario.coletti@snv.jussieu.fr ordario.coletti@uniroma1.it

Tiziana Martinello: tiziana.martinello@unipd.it Marco Patruno: marco.pat@unipd.it

\section{References}

1. Polican Ciena A, Yokomito de Almeida SR, de Matos Alves PH, et al. Histological and ultrastructural changes of sternomastoid muscle in aged wistar rats. Micron 2011;42:871-6.

2. Hiroux C, Vandoorne T, Koppo K,et al. Physical Activity Counteracts Tumor Cell Growth in Colon Carcinoma C26-Injected Muscles: An Interim Report. Eur J Transl Myol 2016;26:5958. doi: 10.4081/ejtm.2016.5958. eCollection 2016 Jun 13.

3. Tasic D, Dimov D, Gligorijevic J, et al. Muscle fibre types and fibre morphometry in the tibialis posterior and anterior of the rat: a comparative study. Medicine and Biology 2003;10:16-21.

4. Coletti D, Daou N, Hassani M, et al. Serum Response Factor in Muscle Tissues: From Development to Ageing. Eur J Transl Myol 2016;26:6008. doi: 10.4081/ejtm.2016.6008. eCollection 2016 Jun 13.

5. Kern H, Loefler S, Hofer C, et al. FES Training in Aging: interim results show statistically significant improvements in mobility and muscle fiber size. Eur J TranslMyology 2012;22:61-7.

6. Brooke MH, Kaiser KK. J. Some comments on the histochemical caracterization of muscle adenosin triphosphatase. Histochem Cytochem 1969;17:4312.

7. Brooke MH, Kaiser KK. Muscle fiber types: How many and what Kind? Arch Neurol 1970 23; 36979.

8. Guth L, Samaha FJ. Qualitative differences between actomyosin ATPase of slow and fast mammalian muscles Exp Neurol 1969;25:139-52.

9. Guth L, Samaha FJ. Procedure for the histochemical demonstration of actomyosin ATPase. Exp Neurol 1970;28: 365-7.
10. Hammalinen N, Pette D. The histochemical profiles of fast fibers IIB, IIA in skeletal muscle of mouse, rat and rabbit. Histochem Cytochem 1993;41:73343.

11. Ciresan DC, Giusti A, Gambardella LM, Schmidhuber J. Mitosis detection in breast cancer histology images with deep neural networks. Med Image Comput Assist Interv 2013;16:411-8.

12. Seene T, Umnova M, Kaasik P. Morphological peculiarities of neuromuscular junctions among different fiber types: Effect of exercise. Eur J Transl Myol. 2017 Jun 27;27(3):6708. doi: 10.4081/ejtm.2017.6708. eCollection 2017 Jun 27.

13. Samiee F, Zarrindast MR. Effect of electrical stimulation on motor nerve regeneration in sciatic nerve ligated-mice. Eur J Transl Myol. 2017 Sep 20;27(3):6488. doi: 10.4081/ejtm.2017.6488. eCollection 2017 Jun 27.

14. Power GA, Dalton BH, Gilmore KJ, et al. Maintaining Motor Units into Old Age: Running the Final Common Pathway. Eur J Transl Myol 2017;27(1):6597. doi: 10.4081/ejtm.2017.6597. eCollection 2017 Feb 24.Pigna E, Greco E, Morozzi $\mathrm{G}$, et al. Denervation does not Induce Muscle Atrophy Through Oxidative Stress. Eur J Transl Myol 2017;27(1):6406. doi: 10.4081/ejtm.2017.6406. eCollection 2017 Feb 24.

15. Pette D, Vrbová G. The Contribution of Neuromuscular Stimulation in Elucidating Muscle Plasticity Revisited. Eur J Transl Myol. 2017 Feb 24;27(1):6368. doi: 10.4081/ejtm.2017.6368. eCollection 2017 Feb 24. Review.

16. Carotenuto F, Coletti D, Di Nardo P, Teodori L. $\alpha$ Linolenic Acid Reduces TNF-Induced Apoptosis in C2C12 Myoblasts by Regulating Expression of Apoptotic Proteins. Eur J Transl Myol 2016;26(4):6033. doi: 10.4081/ejtm.2016.6033. eCollection 2016 Sep 15.22.

17. Riebold B, Nahrstaedt H, Schultheiss C, et al. Multisensor Classification System for Triggering FES in Order to Support Voluntary Swallowing. Eur J Transl Myol 2016;26(4):6224. doi: 10.4081/ejtm.2016.6224. eCollection 2016 Sep 15.33 .

18. Stratton K, Faghri PD. Electrically and HybridInduced Muscle Activations: Effects of Muscle Size and Fiber Type. Eur J Transl Myol 2016;26(3):6163. eCollection 2016 Jun 13.

19. Willand MP. Electrical Stimulation Enhances Reinnervation After Nerve Injury. Eur J Transl Myol 2015;25(4):243-8. doi: 10.4081/ejtm.2015.5243. eCollection 2015 Aug 24. Review.

20. Zampieri S, Mosole S, Löfler S, et al. Physical Exercise in Aging: Nine Weeks of Leg Press or Electrical Stimulation Training in 70 Years Old Sedentary Elderly People. Eur J Transl Myol 


\section{Regional distribution of muscle fiber types in rat sternomastoid muscle}

Eur J Transl Myol 28 (1): 117-123, 2018

2015;25(4):237-42. doi: 10.4081/ejtm.2015.5374. eCollection 2015 Aug 24. Review.

21. Barber L, Scicchitano BM, Musaro A. Molecular and Cellular Mechanisms of Muscle Aging and Sarcopenia and Effects of Electrical Stimulation in Seniors. Eur J Transl Myol 2015;25(4):231-6. doi: 10.4081/ejtm.2015.5227. eCollection 2015 Aug 24. Review.

22. Ravara B, Gobbo V, Carraro U, et al. Functional Electrical Stimulation as a Safe and Effective Treatment for Equine Epaxial Muscle Spasms: Clinical Evaluations and Histochemical Morphometry of Mitochondria in Muscle Biopsies. Eur J Transl Myol 2015;25(2):4910. doi: 10.4081/ejtm.2015.4910. eCollection 2015 Mar 11.

23. Costa A, Rossi E, Scicchitano BM, et al. Neurohypophyseal Hormones: Novel Actors of Striated Muscle Development and Homeostasis. Eur J Transl Myol 2014;24(3):3790. doi: 10.4081/ejtm.2014.3790. eCollection 2014 Sep 23. Review.

24. Hockerman GH, Dethrow NM, Hameed S, et al. The Ubr2 Gene is Expressed in Skeletal Muscle Atrophying as a Result of Hind Limb Suspension, but not Mergla Expression Alone. Eur J Transl Myol. 2014;24(3):3319. doi: 10.4081/ejtm.2014.3319. eCollection 2014 Sep 23.

25. Lomo T. The Response of Denervated Muscle to Long-Term Stimulation (1985, Revisited here in 2014). Eur J Transl Myol 2014;24:3294. doi: 10.4081/ejtm.2014.3294. eCollection 2014 Mar 31.

26. Carlson BM. The Biology of Long-Term Denervated Skeletal Muscle. Eur J Transl Myol. 2014 Mar 27;24:3293. doi: 10.4081/ejtm.2014.3293. eCollection 2014 Mar 31.

Received for publication: January 15, 2018

Revision received: February 13, 2018

Accepted for publication: February 14, 2018 